\title{
Periodic Boundary Value Problems for First-Order Impulsive Functional Integrodifferential Equations with Integral-Jump Conditions
}

\author{
Chatthai Thaiprayoon,, ${ }^{1,2}$ Decha Samana, ${ }^{1,2}$ and Jessada Tariboon ${ }^{2,3}$ \\ ${ }^{1}$ Department of Mathematics, Faculty of Science, King Mongkut's Institute of Technology Ladkrabang, Bangkok 10520, Thailand \\ ${ }^{2}$ Centre of Excellence in Mathematics, CHE, Sri Ayutthaya Road, Bangkok 10400, Thailand \\ ${ }^{3}$ Department of Mathematics, Faculty of Applied Science, King Mongkut's University of Technology North Bangkok, \\ Bangkok 10800, Thailand
}

Correspondence should be addressed to Jessada Tariboon; jessadat@kmutnb.ac.th

Received 6 January 2014; Accepted 11 February 2014; Published 23 March 2014

Academic Editor: Kanishka Perera

Copyright (C) 2014 Chatthai Thaiprayoon et al. This is an open access article distributed under the Creative Commons Attribution License, which permits unrestricted use, distribution, and reproduction in any medium, provided the original work is properly cited.

By developing a new comparison result and using the monotone iterative technique, we are able to obtain existence of minimal and maximal solutions of periodic boundary value problems for first-order impulsive functional integrodifferential equations with integral-jump conditions. An example is also given to illustrate our results.

\section{Introduction}

The theory of impulsive differential equations is now being recognized as not only being richer than the corresponding theory of differential equations without impulses, but also representing a more natural framework for mathematical modelling of many real world phenomena and applications; see $[1-5]$ and the references therein. Monotone iterative technique coupled with the method of upper and lower solutions has provided an effective mechanism to prove constructive existence results for initial and boundary value problems for nonlinear differential equations; see [6]. However, many papers have studied applications of the monotone iterative technique to impulsive problems; see, for example, [7-15]. In those articles, the authors assumed that $\Delta x\left(t_{k}\right)=$ $I_{k}\left(x\left(t_{k}^{-}\right)\right)$, that is, a short-term rapid change of the state (jump condition) at impulse point $t_{k}$, depends on the left side of the limit of $x\left(t_{k}\right)$.

In $[16-18]$ the authors discussed some classes of firstorder impulsive problems with the impulsive integral conditions:

$$
\Delta x\left(t_{k}\right)=I_{k}\left(\int_{t_{k}-\tau_{k}}^{t_{k}} x(s) d s-\int_{t_{k-1}}^{t_{k-1}+\sigma_{k-1}} x(s) d s\right),
$$

where $0<\sigma_{k-1} \leq\left(t_{k}-t_{k-1}\right) / 2$ and $0 \leq \tau_{k} \leq\left(t_{k}-\right.$ $\left.t_{k-1}\right) / 2, k=1,2, \ldots, m$. Furthermore, Thaiprayoon et al. [19] have used such technique to investigate the existence criteria of extremal solutions of multipoint impulsive problems to include multipoint jump conditions

$$
\Delta x\left(t_{k}\right)=I_{k}\left(\sum_{l=1}^{c_{k}} \rho_{l}^{k} x\left(\eta_{l}^{k}\right)\right), \quad c_{k} \in\{1,2, \ldots\},
$$

for $t_{k-1}<\eta_{1}^{k}<\eta_{2}^{k}<\cdots<\eta_{c_{k}}^{k} \leq t_{k}, k=1,2, \ldots, m$. Recently, Thiramanus and Tariboon [20] have given some results on impulsive differential inequalities with integraljump conditions of the form:

$$
\begin{aligned}
x^{\prime}(t) & \leq p(t) m(t)+q(t), \quad t \neq t_{k}, \\
x\left(t_{k}^{+}\right) & \leq d_{k} x\left(t_{k}\right)+c_{k} \int_{t_{k}-\tau_{k}}^{t_{k}-\sigma_{k}} x(s) d s+b_{k}, \quad k=1,2, \ldots,
\end{aligned}
$$

where $0 \leq \sigma_{k} \leq \tau_{k} \leq t_{k}-t_{k-1}$. We note that if $d_{k}=1, \sigma_{k}<\tau_{k}$, $k=1,2, \ldots$, then the above inequalities mean that the bound of jump condition at $t_{k}$ is a functional of past states on the interval $\left(t_{k}-\tau_{k}, t_{k}-\sigma_{k}\right]$ before the impulse point $t_{k}$. 
In spirit of the results from [20], this paper considers the periodic boundary value problem for first-order impulsive functional integrodifferential equation (PBVP) with integraljump conditions:

$$
\begin{aligned}
x^{\prime}(t) & =f(t, x(t), x(\theta(t)),(K x)(t),(S x)(t)), \\
t \in J=[0, T], \quad t \neq t_{k}, & \\
\Delta x\left(t_{k}\right) & =I_{k}\left(\int_{t_{k}-\tau_{k}}^{t_{k}-\sigma_{k}} x(s) d s\right), \quad k=1,2, \ldots, m, \\
x(0) & =x(T),
\end{aligned}
$$

where $0=t_{0}<t_{1}<t_{2}<\cdots<t_{k}<\cdots<t_{m}<t_{m+1}=T, f \in$ $C\left(J \times R^{4}, R\right), \theta \in C(J, J), I_{k} \in C(R, R), \Delta x\left(t_{k}\right)=x\left(t_{k}^{+}\right)-x\left(t_{k}^{-}\right)$, $0 \leq \sigma_{k} \leq \tau_{k} \leq t_{k}-t_{k-1}$,

$$
\begin{aligned}
& (K x)(t)=\int_{0}^{t} k(t, s) x(s) d s \\
& (S x)(t)=\int_{0}^{T} h(t, s) x(s) d s
\end{aligned}
$$

$k(t, s) \in C\left(D, R^{+}\right), h(t, s) \in C\left(J \times J, R^{+}\right), D=\left\{(t, s) \in R^{2}\right.$, $0 \leq s \leq t \leq T\}$, and $R^{+}=[0,+\infty)$.

We first introduce a new concept of lower and upper solutions, then establish a new comparison principle, and discuss the existence and uniqueness of the solutions for firstorder impulsive functional integrodifferential equations with integral-jump conditions. By using the method of upper and lower solutions and monotone iterative technique, we obtain the existence of extreme solution of PBVP (4). Finally, we give an example to illustrate the obtained results.

\section{Preliminaries}

Let $J_{0}=J \backslash\left\{t_{1}, t_{2}, \ldots, t_{m}\right\}, k_{0}=\max \{k(t, s) ;(t, s) \in D\}$, $h_{0}=\max \{h(t, s) ;(t, s) \in J \times J\}$, and $P C(J, R)=\{x:$ $J \rightarrow R ; x(t)$ be continuous everywhere except for some $t_{k}$, at which $x\left(t_{k}^{+}\right)$and $x\left(t_{k}^{-}\right)$exist and $x\left(t_{k}^{-}\right)=x\left(t_{k}\right), k=$ $1,2, \ldots, m\}$, and let $P C^{1}(J, R)=\left\{x \in P C(J, R) ; x^{\prime}(t)\right.$ be continuous everywhere except for some $t_{k}$ at which $x^{\prime}\left(t_{k}^{+}\right)$ and $x^{\prime}\left(t_{k}^{-}\right)$exist and $\left.x^{\prime}\left(t_{k}^{-}\right)=x^{\prime}\left(t_{k}\right), k=1,2, \ldots, m\right\}$. Clearly, $P C(J, R)$ is a Banach space with the norm $\|x\|_{P C}=\sup \{x(t)$ : $t \in J\}$. Let $E=P C(J, R) \cap P C^{1}(J, R)$. A function $x \in E$ is called a solution of PBVP (4) if it satisfies (4).

Definition 1. We say that the functions $\alpha, \beta \in E$ are lower and upper solutions of PBVP (4) if there exist $M>0, W \geq 0$, $N \geq 0, L \geq 0, L_{k} \geq 0$, and $0 \leq \sigma_{k} \leq \tau_{k} \leq t_{k}-t_{k-1}$ such that

$$
\begin{array}{r}
\alpha^{\prime}(t) \leq f(t, \alpha(t), \alpha(\theta(t)),(K \alpha)(t),(S \alpha)(t))-a_{\alpha}(t), \\
t \in J_{0}, \\
\Delta \alpha\left(t_{k}\right) \leq I_{k}\left(\int_{t_{k}-\tau_{k}}^{t_{k}-\sigma_{k}} \alpha(s) d s\right)-b_{\alpha k}, \quad k=1,2, \ldots, m,
\end{array}
$$

where

$$
\begin{aligned}
& a_{\alpha}(t)= \begin{cases}0, & \text { if } \alpha(0) \leq \alpha(T), \\
& \times(T-\theta(t))+1) \times(T)^{-1} \\
+\frac{N \int_{0}^{t} k(t, s)(T-s) d s}{T} & \\
+\frac{L \int_{0}^{T} h(t, s)(T-s) d s}{T} & \\
\times[\alpha(0)-\alpha(T)], & \text { if } \alpha(0)>\alpha(T),\end{cases} \\
& b_{\alpha k}= \begin{cases}0, & \text { if } \alpha(0) \leq \alpha(T), \\
\frac{L_{k}\left(\tau_{k}-\sigma_{k}\right)}{2 T} & \\
\times\left(2\left(T-t_{k}\right)+\tau_{k}+\sigma_{k}\right) & \\
\times[\alpha(0)-\alpha(T)], & \text { if } \alpha(0)>\alpha(T),\end{cases} \\
& \beta^{\prime}(t) \geq f(t, \beta(t), \beta(\theta(t)),(K \beta)(t),(S \beta)(t))+\bar{a}_{\beta}(t), \\
& t \in J_{0} \text {, } \\
& \Delta \beta\left(t_{k}\right) \geq I_{k}\left(\int_{t_{k}-\tau_{k}}^{t_{k}-\sigma_{k}} \beta(s) d s\right)+\bar{b}_{\beta k}, \quad k=1,2, \ldots, m,
\end{aligned}
$$

where

$$
\begin{aligned}
& \bar{a}_{\beta}(t)= \begin{cases}0, & \text { if } \beta(0) \geq \beta(T), \\
(M(T-t)+W & \\
\times(T-\theta(t))+1) \times(T)^{-1} & N \int_{0}^{t} k(t, s)(T-s) d s \\
+\frac{L \int_{0}^{T} h(t, s)(T-s) d s}{T} & \\
\times[\beta(T)-\beta(0)], & \text { if } \beta(0)<\beta(T),\end{cases} \\
& \bar{b}_{\beta k}= \begin{cases}0, & \text { if } \beta(0) \geq \beta(T), \\
\frac{L_{k}\left(\tau_{k}-\sigma_{k}\right)}{2 T} & \\
\times\left(2\left(T-t_{k}\right)+\tau_{k}+\sigma_{k}\right) & \\
\times[\beta(T)-\beta(0)], & \text { if } \beta(0)<\beta(T) .\end{cases}
\end{aligned}
$$

Denote $l=\max \left\{k: t \geq t_{k}, \mathrm{k}=1,2, \ldots\right\}$. We prove the comparison principle by using the following lemma (see [20]). 
Lemma 2. Let $r \in\left\{t_{0}, t_{1}, \ldots, t_{m}\right\}, c_{k} \geq 0$, and $0 \leq \sigma_{k} \leq \tau_{k} \leq$ $t_{k}-t_{k-1}, \gamma_{k}, k=1, \ldots, m$, be constants and let $q \in P C(J, R)$, $x \in P C^{1}(J, R)$. If

$$
\begin{aligned}
x^{\prime}(t) & \geq q(t), \quad t \in(r, T), \quad t \neq t_{k}, \\
\Delta x\left(t_{k}\right) & \geq c_{k} \int_{t_{k}-\tau_{k}}^{t_{k}-\sigma_{k}} x(s) d s+\gamma_{k}, \quad t_{k} \in(r, T),
\end{aligned}
$$

then, for $t \in(r, T]$,

$$
\begin{aligned}
x(t) \geq x\left(r^{+}\right)\left(\prod_{r<t_{k}<t}\left[1+c_{k}\left(\tau_{k}-\sigma_{k}\right)\right]\right) \\
+\sum_{r<t_{k}<t}\left[\prod_{t_{k}<t_{j}<t}\left(1+c_{j}\left(\tau_{j}-\sigma_{j}\right)\right)\right. \\
\quad \times\left(\left[1+c_{k}\left(\tau_{k}-\sigma_{k}\right)\right] \int_{t_{k-1}}^{t_{k}-\tau_{k}} q(s) d s\right. \\
+\int_{t_{k}-\tau_{k}}^{t_{k}-\sigma_{k}}\left[1+c_{k}\left(t_{k}-\sigma_{k}-s\right)\right] q(s) d s \\
\left.\left.+\int_{t_{k}-\sigma_{k}}^{t_{k}} q(s) d s+\gamma_{k}\right)\right]+\int_{t_{l}}^{t} q(s) d s .
\end{aligned}
$$

Now we are in the position to establish a new comparison principle, which plays an important role in monotone iterative technique.

\section{Lemma 3. Assume that $x \in E$ satisfies}

$$
\begin{aligned}
x^{\prime}(t) \geq & M x(t)+W x(\theta(t))+N \int_{0}^{t} k(t, s) x(s) d s \\
& +L \int_{0}^{T} h(t, s) x(s) d s+\bar{a}_{x}(t), \quad t \in J_{0}, \\
\Delta x\left(t_{k}\right) \geq & L_{k} \int_{t_{k}-\tau_{k}}^{t_{k}-\sigma_{k}} x(s) d s+\bar{b}_{x k}, \quad k=1,2, \ldots, m,
\end{aligned}
$$

where constants $M>0, W \geq 0, N \geq 0, L \geq 0, L_{k} \geq 0$, and $0 \leq \sigma_{k} \leq \tau_{k} \leq t_{k}-t_{k-1}, k=1,2, \ldots$, m. If

$$
\left(\widehat{L}+\sum_{k=1}^{m} B_{k}+\int_{0}^{T} r(s) d s\right) \prod_{k=1}^{m} A_{k} \leq 1,
$$

where $\bar{a}_{x}(t), t \in J_{0}$, and $\bar{b}_{x k}$ are given by Definition 1 with $\beta=x$ and

$$
\begin{aligned}
\widehat{L} & =\max \left\{L_{k}\left(\tau_{k}-\sigma_{k}\right) ; k=1,2, \ldots, m\right\} \\
r(t) & =M+W+N \int_{0}^{t} k(t, s) d s+L \int_{0}^{T} h(t, s) d s \\
A_{k} & =1+L_{k}\left(\tau_{k}-\sigma_{k}\right)
\end{aligned}
$$

$$
\begin{aligned}
B_{k}=A_{k} & \int_{t_{k-1}}^{t_{k}-\tau_{k}} r(s) d s \\
& +\int_{t_{k}-\tau_{k}}^{t_{k}-\sigma_{k}}\left[1+L_{k}\left(t_{k}-\sigma_{k}-s\right)\right] r(s) d s \\
& +\int_{t_{k}-\sigma_{k}}^{t_{k}} r(s) d s,
\end{aligned}
$$

then $x(t) \leq 0, t \in J$.

Proof.

Case 1. One has $x(0) \geq x(\mathrm{~T})$. Suppose that there exists $t^{*} \in J$ such that $x\left(t^{*}\right)>0$ and distinguish two cases.

Case (a). $x(t) \geq 0$ for all $t \in J, x \neq \equiv$; then

$$
\begin{gathered}
x^{\prime}(t) \geq M x(t)+W x(\theta(t))+N \int_{0}^{t} k(t, s) x(s) d s \\
+L \int_{0}^{T} h(t, s) x(s) d s \geq 0, \quad t \in J_{0}, \\
x\left(t_{k}^{+}\right) \geq x\left(t_{k}\right)+L_{k} \int_{t_{k}-\tau_{k}}^{t_{k}-\sigma_{k}} x(s) d s \geq x\left(t_{k}\right), \\
k=1,2, \ldots, m,
\end{gathered}
$$

so that $x$ is nondecreasing in $J$, and then $x(T) \geq x(0)$. Since $x(0) \geq x(T)$, then $x$ is a constant function $x(t)=C>0$, which implies that

$$
\begin{aligned}
x^{\prime}(t)= & 0 \geq M C+W C+N C \int_{0}^{t} k(t, s) d s \\
& +L C \int_{0}^{T} h(t, s) d s \geq M C>0,
\end{aligned}
$$

getting a contradiction.

Case (b). $x(t)<0$ for some $t \in J$. Let $\inf _{t \in J} x(t)=-\lambda<0$; then there exists $\bar{t} \in\left(t_{i}, t_{i+1}\right]$, for some $i$ such that $x(\bar{t})=-\lambda$ or $x\left(t_{i}^{+}\right)=-\lambda$. Without loss of generality, we only consider $x(\bar{t})=-\lambda$, and for the case $x\left(t_{i}^{+}\right)=-\lambda$ the proof is similar.

From (11), it is easy to see that

$$
\begin{aligned}
x^{\prime}(t) \geq-\lambda(M & +W+N \int_{0}^{t} k(t, s) d s \\
& \left.+L \int_{0}^{T} h(t, s) d s\right), \quad t \in J_{0} .
\end{aligned}
$$

We consider the inequalities

$$
\begin{aligned}
x^{\prime}(t) & \geq-\lambda r(t), \quad t \in J_{0}, \\
\Delta x\left(t_{k}\right) & \geq L_{k} \int_{t_{k}-\tau_{k}}^{t_{k}-\sigma_{k}} x(s) d s, \quad k=1,2, \ldots, m .
\end{aligned}
$$


From Lemma 2, we have

$$
\begin{aligned}
x(t) \geq x(0) & \prod_{0<t_{k}<t} A_{k} \\
& -\lambda\left[\sum_{0<t_{k}<t}\left(\prod_{t_{k}<t_{j}<t} A_{j} B_{k}\right)+\int_{t_{l}}^{t} r(s) d s\right],
\end{aligned}
$$$$
t \in J \text {. }
$$

Let $t=\bar{t}$ in (18); then

$$
\begin{aligned}
x(\bar{t}) \geq & x(0) \prod_{0<t_{k}<\bar{t}} A_{k} \\
& -\lambda\left[\sum_{0<t_{k}<\bar{t}}\left(\prod_{t_{k}<t_{j}<\bar{t}} A_{j} B_{k}\right)+\int_{t_{i}}^{\bar{t}} r(s) d s\right],
\end{aligned}
$$

so that

$$
\begin{aligned}
x(0) \prod_{0<t_{k}<\bar{t}} A_{k} \leq & -\lambda \\
& +\lambda\left[\sum_{0<t_{k}<\bar{t}}\left(\prod_{t_{k}<t_{j}<\bar{t}} A_{j} B_{k}\right)+\int_{t_{i}}^{\bar{t}} r(s) d s\right] .
\end{aligned}
$$

If $x(0)>0$, then (20) with $A_{k} \geq 1, B_{k} \geq 0$ for all $k$ implies

$$
\begin{aligned}
1 & <\sum_{0<t_{k}<\bar{t}}\left(\prod_{t_{k}<t_{j}<\bar{t}} A_{j} B_{k}\right)+\int_{t_{i}}^{\bar{t}} r(s) d s \\
& \leq \sum_{k=1}^{m}\left(\prod_{k<j<m} A_{j} B_{k}\right)+\int_{0}^{T} r(s) d s .
\end{aligned}
$$

This contradicts the condition (12).

Suppose that $x(0) \leq 0$. If $t^{*}<\bar{t}$ for $t^{*} \in\left(t_{\nu}, t_{\nu+1}\right]$, then Lemma 2 provides that

$$
\begin{array}{r}
x(t) \geq x\left(t_{\nu+1}^{+}\right) \prod_{t_{\nu+1}<t_{k}<t} A_{k} \\
-\lambda\left[\sum_{t_{\nu+1}<t_{k}<t}\left(\prod_{t_{k}<t_{j}<t} A_{j} B_{k}\right)+\int_{t_{l}}^{t} r(s) d s\right], \\
t \geq t_{\nu+1}^{+} .
\end{array}
$$

Since

$$
\begin{gathered}
x\left(t_{\nu+1}^{+}\right) \geq x\left(t_{\nu+1}\right)+L_{\nu+1} \int_{t_{\nu+1}-\tau_{\nu+1}}^{t_{\nu+1}-\sigma_{\nu+1}} x(s) d s \\
\geq x\left(t_{\nu+1}\right)-\lambda L_{\nu+1}\left(\tau_{\nu+1}-\sigma_{\nu+1}\right),
\end{gathered}
$$

and integrating (11) from $t^{*}$ into $t_{\nu+1}$, we obtain

$$
x\left(t_{\nu+1}\right) \geq x\left(t^{*}\right)-\lambda \int_{t^{*}}^{t_{\nu+1}} r(s) d s .
$$

Hence,

$x(t)$

$$
\begin{aligned}
\geq & x\left(t^{*}\right)\left(\prod_{t_{v+1}<t_{k}<t} A_{k}\right)-\lambda L_{\nu+1}\left(\tau_{v+1}-\sigma_{\nu+1}\right) \\
& \times\left(\prod_{t_{v+1}<t_{k}<t} A_{k}\right)-\lambda\left(\prod_{t_{\nu+1}<t_{k}<t} A_{k}\right) \int_{t^{*}}^{t_{v+1}} r(s) d s \\
& -\lambda\left[\sum_{t_{v+1}<t_{k}<t}\left(\prod_{t_{k}<t_{j}<t} A_{j} B_{k}\right)+\int_{t_{l}}^{t} r(s) d s\right] \\
\geq & x\left(t^{*}\right)\left(\prod_{t_{v+1}<t_{k}<t} A_{k}\right)-\lambda L_{v+1}\left(\tau_{\nu+1}-\sigma_{\nu+1}\right)\left(\prod_{t_{v+1}<t_{k}<t} A_{k}\right) \\
& -\lambda\left[\sum_{t_{v}<t_{k}<t}\left(\prod_{t_{k}<t_{j}<t} A_{j} B_{k}\right)+\int_{t_{l}}^{t} r(s) d s\right], \quad t \geq t^{*} .
\end{aligned}
$$

We note that if $t \in\left(t_{v}, t_{v+1}\right]$, then $t_{l}=t^{*}$.

$$
\text { Let } t=\bar{t} \text { in (25); then }
$$

$x(\bar{t})$

$$
\begin{aligned}
& \geq x\left(t^{*}\right)\left(\prod_{t_{v+1}<t_{k}<\bar{t}} A_{k}\right)-\lambda L_{\nu+1}\left(\tau_{\nu+1}-\sigma_{\nu+1}\right)\left(\prod_{t_{v+1}<t_{k}<\bar{t}} A_{k}\right) \\
& \quad-\lambda\left[\sum_{t_{v}<t_{k}<\bar{t}}\left(\prod_{t_{k}<t_{j}<\bar{t}} A_{j} B_{k}\right)+\int_{t_{l}}^{\bar{t}} r(s) d s\right] .
\end{aligned}
$$

From (26), we have

$$
\begin{aligned}
0<x\left(t^{*}\right)\left(\prod_{t_{v+1}<t_{k}<\bar{t}} A_{k}\right) \\
\leq-\lambda+\lambda L_{\nu+1}\left(\tau_{\nu+1}-\sigma_{\nu+1}\right)\left(\prod_{t_{\nu+1}<t_{k}<\bar{t}} A_{k}\right) \\
+\lambda\left[\sum_{t_{\nu}<t_{k}<\bar{t}}\left(\prod_{t_{k}<t_{j}<\bar{t}} A_{j} B_{k}\right)+\int_{t_{l}}^{\bar{t}} r(s) d s\right],
\end{aligned}
$$

which gives

$$
1<\widehat{L} \prod_{k=1}^{m} A_{k}+\sum_{0<t_{k}<T}\left(\prod_{t_{k}<t_{j}<T} A_{j} B_{k}\right)+\int_{0}^{T} r(s) d s,
$$


contradicting condition (12). For the case $\bar{t}<t^{*}$, the proof is similar.

Case 2. One has $x(0)<x(T)$. Set $v(t)=x(t)+((T-$ $t) / T)(x(T)-x(0))$. It follows that $v(0)=x(T)=v(T)$, and for $t \in J_{0}$,

$$
\begin{aligned}
v^{\prime}(t) \geq & M x(t)+W x(\theta(t))+N \int_{0}^{t} k(t, s) x(s) d s \\
& +L \int_{0}^{T} h(t, s) x(s) d s+\bar{a}_{x}(t)-\frac{1}{T}(x(T)-x(0)) \\
= & M v(t)+W v(\theta(t))+N \int_{0}^{t} k(t, s) v(s) d s \\
& +L \int_{0}^{T} h(t, s) v(s) d s+\bar{a}_{x}(t) \\
& -\frac{M(T-t)+W(T-\theta(t))+1}{T}[x(T)-x(0)] \\
& -\frac{N[x(T)-x(0)]}{T} \int_{0}^{t} k(t, s)(T-s) d s \\
& -\frac{L[x(T)-x(0)]}{T} \int_{0}^{t} h(t, s)(T-s) d s \\
\geq & M v(t)+W v(\theta(t))+N \int_{0}^{t} k(t, s) v(s) d s \\
& +L \int_{0}^{T} h(t, s) v(s) d s,
\end{aligned}
$$

and for $t=t_{k}, k=1,2, \ldots, m$,

$$
\begin{aligned}
\Delta v\left(t_{k}\right)= & \Delta x\left(t_{k}\right) \geq L_{k} \int_{t_{k}-\tau_{k}}^{t_{k}-\sigma_{k}} x(s) d s \\
& +\frac{L_{k}\left(\tau_{k}-\sigma_{k}\right)\left[2\left(T-t_{k}\right)+\tau_{k}+\sigma_{k}\right]}{2 T} \\
& \times(x(T)-x(0))=L_{k} \int_{t_{k}-\tau_{k}}^{t_{k}-\sigma_{k}} v(s) d s .
\end{aligned}
$$

In view of Case 1 , we see that $v(t) \leq 0$ on $J$, and therefore $x(t) \leq 0$ on $J$. This completes the proof.

\section{Corollary 4. Assume that $x \in E$ satisfies}

$$
\begin{array}{r}
x^{\prime}(t) \geq M x(t)+W x(\theta(t))+N \int_{0}^{t} k(t, s) x(s) d s \\
+L \int_{0}^{T} h(t, s) x(s) d s, \quad t \in J_{0}, \\
\Delta x\left(t_{k}\right) \geq L_{k} \int_{t_{k}-\tau_{k}}^{t_{k}-\sigma_{k}} x(s) d s, \quad k=1,2, \ldots, m, \\
x(0) \geq x(T),
\end{array}
$$

where constants $M>0, W \geq 0, N \geq 0, L \geq 0, L_{k} \geq 0$, and $0 \leq \sigma_{k} \leq \tau_{k} \leq t_{k}-t_{k-1}, k=1,2, \ldots$, m. If

$$
\left(\widehat{L}+\sum_{k=1}^{m} B_{k}^{0}+r_{0} T\right) \prod_{k=1}^{m} A_{k} \leq 1,
$$

where

$$
\begin{aligned}
r_{0}= & M+W+k_{0} N T+h_{0} L T \\
B_{k}^{0}=r_{0} A_{k} \int_{t_{k-1}}^{t_{k}-\tau_{k}} d s & \\
& +r_{0} \int_{t_{k}-\tau_{k}}^{t_{k}-\sigma_{k}}\left[1+L_{k}\left(t_{k}-\sigma_{k}-s\right)\right] d s+r_{0} \int_{t_{k}-\sigma_{k}}^{t_{k}} d s \\
= & r_{0}\left[\left(t_{k}-t_{k-1}\right)+\frac{L_{k}}{2}\left(\tau_{k}-\sigma_{k}\right)\right. \\
& \left.\quad \times\left(2\left(t_{k}-t_{k-1}\right)-\left(\tau_{k}+\sigma_{k}\right)\right)\right],
\end{aligned}
$$

and $\widehat{L}, A_{k}$ are given by Lemma 3 , then $x(t) \leq 0$, for $t \in J$.

Corollary 5. Assume that $x \in E$ satisfies

$$
\begin{aligned}
x^{\prime}(t) & \\
\geq & M x(t)+W x(\theta(t)) \\
& +N \int_{0}^{t} k(t, s) x(s) d s+L \int_{0}^{T} h(t, s) x(s) d s \\
& +\left(\frac{M(T-t)+W(T-\theta(t))+1}{T}\right. \\
& +\frac{k_{0} N\left(2 T t-t^{2}\right)}{2 T} \\
& +\frac{\left.h_{0} L T\right)[x(T)-x(0)], \quad t \in J_{0},}{2} \quad \\
\Delta x\left(t_{k}\right) \geq & L_{k} \int_{t_{k}-\tau_{k}}^{t_{k}-\sigma_{k}} x(s) d s \\
& +\frac{L_{k}\left(\tau_{k}-\sigma_{k}\right)\left[2\left(T-t_{k}\right)+\tau_{k}+\sigma_{k}\right]}{2 T} \quad x(0)<x(T), \\
& \times(x(T)-x(0)), \quad k=1,2, \ldots, m, \\
&
\end{aligned}
$$

where constants $M>0, W \geq 0, N \geq 0, L \geq 0, L_{k} \geq 0$, and $0 \leq \sigma_{k} \leq \tau_{k} \leq t_{k}-t_{k-1}, k=1,2, \ldots, m$, and condition (32) holds. Then $x(t) \leq 0$, for $t \in J$. 
Let us consider the following linear problem of PBVP (4):

$$
\begin{aligned}
x^{\prime}(t)-M x(t)= & W x(\theta(t))+N \int_{0}^{t} k(t, s) x(s) d s \\
& +L \int_{0}^{T} h(t, s) x(s) d s+g(t), \quad t \in J_{0}, \\
\Delta x\left(t_{k}\right)= & L_{k} \int_{t_{k}-\tau_{k}}^{t_{k}-\sigma_{k}} x(s) d s+I_{k}\left(\int_{t_{k}-\tau_{k}}^{t_{k}-\sigma_{k}} \eta(s) d s\right) \\
& -L_{k} \int_{t_{k}-\tau_{k}}^{t_{k}-\sigma_{k}} \eta(s) d s, \quad k=1,2, \ldots, m, \\
& x(0)=x(T),
\end{aligned}
$$

where $M>0, W \geq 0, N \geq 0, L \geq 0, L_{k} \geq 0,0 \leq \sigma_{k} \leq \tau_{k} \leq$ $t_{k}-t_{k-1}, \theta \in(J, J), I_{k} \in C(J, R), k=1,2, \ldots, m, g \in P C(J, R)$, and $\eta \in P C^{1}(J, R)$.

Lemma 6. A function $x \in E$ is a solution of (35) if and only if $x \in P C(J, R)$ is a solution of the following impulsive integral equation:

$$
\begin{aligned}
x(t)=- & \int_{0}^{T} G(t, s) R(s) d s-\sum_{k=1}^{m} G\left(t, t_{k}\right) \\
\times & {\left[L_{k} \int_{t_{k}-\tau_{k}}^{t_{k}-\sigma_{k}} x(s) d s+I_{k}\left(\int_{t_{k}-\tau_{k}}^{t_{k}-\sigma_{k}} \eta(s) d s\right)\right.} \\
& \left.-L_{k} \int_{t_{k}-\tau_{k}}^{t_{k}-\sigma_{k}} \eta(s) d s\right], \quad t \in J,
\end{aligned}
$$

where $R(t)=W x(\theta(t))+N \int_{0}^{t} k(t, s) x(s) d s+$ $L \int_{0}^{T} h(t, s) x(s) d s+g(t)$ and

$$
G(t, s)=\frac{1}{e^{M T}-1} \begin{cases}e^{M(t-s)}, & 0 \leq s<t \leq T \\ e^{M(T+t-s)}, & 0 \leq t \leq s \leq T\end{cases}
$$

Proof. If $x(t)$ is a solution of (35), by directly integrating, we obtain

$$
\begin{aligned}
x(t)=- & \int_{0}^{T} G(t, s) R(s) d s-\sum_{k=1}^{m} G\left(t, t_{k}\right) \\
\times & {\left[L_{k} \int_{t_{k}-\tau_{k}}^{t_{k}-\sigma_{k}} x(s) d s+I_{k}\left(\int_{t_{k}-\tau_{k}}^{t_{k}-\sigma_{k}} \eta(s) d s\right)\right.} \\
& \left.-L_{k} \int_{t_{k}-\tau_{k}}^{t_{k}-\sigma_{k}} \eta(s) d s\right], \quad t \in J .
\end{aligned}
$$

If $x(t)$ is a solution of the above-mentioned integral equation, then

$$
\begin{aligned}
& x^{\prime}(t)=M\left\{-\int_{0}^{t} \frac{e^{M(t-s)}}{e^{M T}-1} R(s) d s-\int_{t}^{T} \frac{e^{M(T+t-s)}}{e^{M T}-1} R(s) d s\right. \\
& -\sum_{0<t_{k}<t} \frac{e^{M\left(t-t_{k}\right)}}{e^{M T}-1}\left[L_{k} \int_{t_{k}-\tau_{k}}^{t_{k}-\sigma_{k}} x(s) d s\right. \\
& +\mathrm{I}_{k}\left(\int_{t_{k}-\tau_{k}}^{t_{k}-\sigma_{k}} \eta(s) d s\right) \\
& \left.-L_{k} \int_{t_{k}-\tau_{k}}^{t_{k}-\sigma_{k}} \eta(s) d s\right] \\
& -\sum_{t \leq t_{k}<t} \frac{e^{M\left(T+t-t_{k}\right)}}{e^{M T}-1}\left[L_{k} \int_{t_{k}-\tau_{k}}^{t_{k}-\sigma_{k}} x(s) d s\right. \\
& +I_{k}\left(\int_{t_{k}-\tau_{k}}^{t_{k}-\sigma_{k}} \eta(s) d s\right) \\
& \left.\left.-L_{k} \int_{t_{k}-\tau_{k}}^{t_{k}-\sigma_{k}} \eta(s) d s\right]\right\} \\
& +R(t) \\
& =M\left\{-\int_{0}^{T} G(t, s) R(s) d s\right. \\
& -\sum_{k=1}^{m} G\left(t, t_{k}\right)\left[L_{k} \int_{t_{k}-\tau_{k}}^{t_{k}-\sigma_{k}} x(s) d s\right. \\
& +I_{k}\left(\int_{t_{k}-\tau_{k}}^{t_{k}-\sigma_{k}} \eta(s) d s\right) \\
& \left.\left.-L_{k} \int_{t_{k}-\tau_{k}}^{t_{k}-\sigma_{k}} \eta(s) d s\right]\right\}+R(t) \\
& =M x(t)+R(t), \\
& \Delta x\left(t_{k}\right)=x\left(t_{k}^{+}\right)-x\left(t_{k}^{-}\right) \\
& =\frac{e^{M T}}{e^{M T}-1}\left[L_{k} \int_{t_{k}-\tau_{k}}^{t_{k}-\sigma_{k}} x(s) d s+I_{k}\left(\int_{t_{k}-\tau_{k}}^{t_{k}-\sigma_{k}} \eta(s) d s\right)\right. \\
& \left.-L_{k} \int_{t_{k}-\tau_{k}}^{t_{k}-\sigma_{k}} \eta(s) d s\right] \\
& -\frac{1}{e^{M T}-1}\left[L_{k} \int_{t_{k}-\tau_{k}}^{t_{k}-\sigma_{k}} x(s) d s\right. \\
& +I_{k}\left(\int_{t_{k}-\tau_{k}}^{t_{k}-\sigma_{k}} \eta(s) d s\right) \\
& \left.-L_{k} \int_{t_{k}-\tau_{k}}^{t_{k}-\sigma_{k}} \eta(s) d s\right] \\
& x(0)=-\int_{0}^{T} \frac{e^{M(T-s)}}{e^{M T}-1} R(s) d s+\sum_{k=1}^{m} \frac{e^{M\left(T-t_{k}\right)}}{e^{M T}-1}
\end{aligned}
$$




$$
\begin{array}{r}
\times\left[L_{k} \int_{t_{k}-\tau_{k}}^{t_{k}-\sigma_{k}} x(s) d s+I_{k}\left(\int_{t_{k}-\tau_{k}}^{t_{k}-\sigma_{k}} \eta(s) d s\right)\right. \\
\left.-L_{k} \int_{t_{k}-\tau_{k}}^{t_{k}-\sigma_{k}} \eta(s) d s\right]=x(T) .
\end{array}
$$

The proof is complete.

Lemma 7. Let $M>0, W \geq 0, N \geq 0, L \geq 0, L_{k} \geq 0$, $0 \leq \sigma_{k} \leq \tau_{k} \leq t_{k}-t_{k-1}, \theta \in(J, J), I_{k} \in C(J, R), k=1,2, \ldots, m$, $g \in P C(J, R)$, and $\eta \in P C^{1}(J, R)$ and assume that

$$
\begin{aligned}
& \sup _{t \in J}\left\{\int_{0}^{T} G(t, s)(W\right.+N \int_{0}^{s} k(s, r) d r \\
&\left.\left.+L \int_{0}^{T} h(s, r) d r\right) d s\right\} \\
&+\frac{1}{1-e^{-M T}} \sum_{k=1}^{m} L_{k}\left(\tau_{k}-\sigma_{k}\right)<1 .
\end{aligned}
$$

Then problem (35) has a unique solution in $P C(J, R)$.

Proof. We define the mapping $F: P C(J, R) \rightarrow P C(J, R)$ by

$$
\begin{aligned}
(F x)(t)=-\int_{0}^{T} G(t, s)( & W x(\theta(s))+N \int_{0}^{s} k(s, r) x(r) d r \\
& \left.+L \int_{0}^{T} h(s, r) x(r) d r+g(s)\right) d s \\
-\sum_{k=1}^{m} G\left(t, t_{k}\right)( & L_{k} \int_{t_{k}-\tau_{k}}^{t_{k}-\sigma_{k}} x(s) d s \\
& +I_{k}\left(\int_{t_{k}-\tau_{k}}^{t_{k}-\sigma_{k}} \eta(s) d s\right) \\
& \left.-L_{k} \int_{t_{k}-\tau_{k}}^{t_{k}-\sigma_{k}} \eta(s) d s\right), \quad t \in J,
\end{aligned}
$$

for any $x \in P C(J, R)$ and $G$ is given by Lemma 6 . Then

$$
\begin{aligned}
& \|F x-F y\|_{P C} \\
& =\sup _{t \in J} \mid-\int_{0}^{T} G(t, s)(W[x(\theta(s))-y(\theta(s))] \\
& +N \int_{0}^{s} k(s, r)[x(r)-y(r)] d r \\
& \left.+L \int_{0}^{T} h(s, r)[x(r)-y(r)] d r\right) d s
\end{aligned}
$$

$$
\begin{gathered}
-\sum_{k=1}^{m} G\left(t, t_{k}\right)\left(L_{k} \int_{t_{k}-\tau_{k}}^{t_{k}-\sigma_{k}}[x(s)-y(s)] d s\right) \mid \\
\leq \sup _{t \in J}\left\{\int_{0}^{T} G(t, s)(W|x(\theta(s))-y(\theta(s))|\right. \\
+N \int_{0}^{s} k(s, r)|x(r)-y(r)| d r \\
\left.+L \int_{0}^{T} h(s, r)|x(r)-y(r)| d r\right) d s \\
\left.+\|x-y\|_{P C}^{m} G\left(t, t_{k}\right)\left(L_{k} \int_{t_{k}-\tau_{k}}^{t_{k}-\sigma_{k}}|x(r)-y(r)| d s\right)\right\} \\
\times\left[\operatorname { s u p } _ { t \in J } \left\{\int_{0}^{T} G(t, s)\right.\right. \\
\left.\quad \times\left(W+N \int_{0}^{s} k(s, r) d r+L \int_{0}^{T} h(s, r) d r\right) d s\right\} \\
\left.+\frac{1}{1-e^{-M T}} \sum_{k=1}^{m} L_{k}\left(\tau_{k}-\sigma_{k}\right)\right]
\end{gathered}
$$

The above result and condition (40) imply that $F$ is a contractive mapping, which completes the proof.

Corollary 8. Let $M>0, W \geq 0, N \geq 0, L \geq 0, L_{k} \geq 0$, $0 \leq \sigma_{k} \leq \tau_{k} \leq t_{k}-t_{k-1}, \theta \in(J, J), I_{k} \in C(J, R), k=1,2, \ldots, m$, $g \in P C(J, R)$, and $\eta \in P C^{1}(J, R)$ and assume that

$$
\frac{W+\left(k_{0} N+h_{0} L\right) T}{M}+\frac{1}{1-e^{-M T}} \sum_{k=1}^{m} L_{k}\left(\tau_{k}-\sigma_{k}\right)<1 .
$$

Then problem (35) has a unique solution in $\operatorname{PC}^{1}(J, R)$.

\section{Main Results}

In this section, we establish existence criteria for solutions of the PBVP (4) by the method of lower and upper solutions and monotone iterative technique. For $\alpha, \beta \in E$, we write $\beta \leq \alpha$ if $\beta(t) \leq \alpha(t)$ for all $t \in J$. In such a case, we denote $[\beta, \alpha]=$ $\{x \in E: \beta(t) \leq x(t) \leq \alpha(t), t \in J\}$.

Theorem 9. Assume the existence of lower and upper solutions for PBVP (4) and also suppose that the following conditions hold.

$\left(H_{1}\right)$ The function $f \in C\left(J \times R^{4}, R\right)$ satisfies

$$
\begin{gathered}
f\left(t, z_{1}, z_{2}, z_{3}, z_{4}\right)-f\left(t, y_{1}, y_{2}, y_{3}, y_{4}\right) \\
\leq M\left(z_{1}-y_{1}\right)+W\left(z_{2}-y_{2}\right) \\
\quad+N\left(z_{3}-y_{3}\right)+L\left(z_{4}-y_{4}\right), \\
f\left(t, y_{1}, y_{2}, y_{3}, y_{4}\right)-f\left(t, x_{1}, x_{2}, x_{3}, x_{4}\right)
\end{gathered}
$$




$$
\begin{aligned}
\leq & M\left(y_{1}-x_{1}\right)+W\left(y_{2}-x_{2}\right) \\
& +N\left(y_{3}-x_{3}\right)+L\left(y_{4}-x_{4}\right),
\end{aligned}
$$

where $\beta(t) \leq x_{1} \leq y_{1} \leq z_{1} \leq \alpha(t), \beta(\theta(t)) \leq x_{2} \leq y_{2} \leq z_{2} \leq$ $\alpha(\theta(t)),(K \beta)(t) \leq x_{3} \leq y_{3} \leq z_{3} \leq(K \alpha)(t)$, and $(S \beta)(t) \leq$ $x_{4} \leq y_{4} \leq z_{4} \leq(S \alpha)(t), t \in J$, where $M>0, W \geq 0, N \geq 0$, $L \geq 0$, and $\theta \in C(J, J)$.

$\left(H_{2}\right)$ The functions $I_{k} \in C(R, R)$ satisfy

$$
\begin{aligned}
& I_{k}\left(\int_{t_{k}-\tau_{k}}^{t_{k}-\sigma_{k}} \alpha(s) d s\right)-I_{k}\left(\int_{t_{k}-\tau_{k}}^{t_{k}-\sigma_{k}} y(s) d s\right) \\
& \quad \leq L_{k} \int_{t_{k}-\tau_{k}}^{t_{k}-\sigma_{k}} \alpha(s)-y(s) d s, \\
& I_{k}\left(\int_{t_{k}-\tau_{k}}^{t_{k}-\sigma_{k}} y(s) d s\right)-I_{k}\left(\int_{t_{k}-\tau_{k}}^{t_{k}-\sigma_{k}} \beta(s) d s\right) \\
& \quad \leq L_{k} \int_{t_{k}-\tau_{k}}^{t_{k}-\sigma_{k}} y(s)-\beta(s) d s,
\end{aligned}
$$

where $\int_{t_{k}-\tau_{k}}^{t_{k}-\sigma_{k}} \beta(s) d s \leq \int_{t_{k}-\tau_{k}}^{t_{k}-\sigma_{k}} y(s) d s \leq \int_{t_{k}-\tau_{k}}^{t_{k}-\sigma_{k}} \alpha(s) d s, k=$ $1,2, \ldots, m$, where $L_{k} \geq 0$ and $0 \leq \sigma_{k} \leq \tau_{k} \leq t_{k}-t_{k-1}$, $k=1,2, \ldots, m$.

If inequalities (12) and (40) hold, then there exists a solution $x$ of $P B V P(4)$ such that $\beta(t) \leq x(t) \leq \alpha(t)$, for $t \in J$.

Proof. We consider the following modified problem relative to PBVP (4):

$$
\begin{aligned}
& x^{\prime}(t)-M x(t)= W x(\theta(t))+N(K x)(t) \\
&+L(S x)(t)+g_{q}(t), \quad t \in J_{0}, \\
& \Delta x\left(t_{k}\right)= L_{k} \int_{t_{k}-\tau_{k}}^{t_{k}-\sigma_{k}} x(s) d s \\
&+I_{k}\left(\int_{t_{k}-\tau_{k}}^{t_{k}-\sigma_{k}} q(s, x(s)) d s\right) \\
&-L_{k} \int_{t_{k}-\tau_{k}}^{t_{k}-\sigma_{k}} q(s, x(s)) d s, \quad k=1,2, \ldots, m, \\
& x(0)=x(T),
\end{aligned}
$$

where $g_{q}(t)=f(t, q(t), q(\theta(t)),(K q)(t),(S q)(t))-M q(t)-$ $W q(\theta(t))-N(K q)(t)-L(S q)(t)$ and

$$
\begin{aligned}
q(t, x(t)) & =\max \{\beta(t), \min \{x(t), \alpha(t)\}\} \\
& = \begin{cases}\beta(t), & \text { if } x<\beta(t), \\
x(t), & \text { if } \beta(t) \leq x \leq \alpha(t), \\
\alpha(t), & \text { if } x>\alpha(t) .\end{cases}
\end{aligned}
$$

If $x \in E$ is such that $\beta \leq x \leq \alpha$ on $J$, then $x$ is a solution of PBVP (4) if and only if $x$ is a solution of (46). We will show that (46) is solvable and that every solution of (46) satisfies $\beta \leq x \leq \alpha$ on $J$. Suppose that $x \in E$ is a solution of (46). We will show that $\beta \leq x$. Let $p=\beta-x$. Then, we have $p(0)-$ $p(T)=\beta(0)-\beta(T)$ since $x(0)=x(T)$ and

$$
\begin{aligned}
p^{\prime}(t)= & \beta^{\prime}(t)-x^{\prime}(t) \\
\geq & f(t, \beta(t), \beta(\theta(t)),(K \beta)(t),(S \beta)(t)) \\
& +\bar{a}_{\beta}-[M x(t)+W x(\theta(t))+N(K x)(t) \\
& +L(S x)(t) \\
& +f(t, q(t), q(\theta(t)),(K q)(t),(S q)(t)) \\
& -M q(t)-W q(\theta(t)) \\
\geq & M p(t)+W p(\theta(t))+N(K p)(t) \\
& +L(S p)(t)+\bar{a}_{p}(t), \quad t \in J_{0} . \\
\Delta p\left(t_{k}\right)= & \Delta \beta\left(t_{k}\right)-\Delta x\left(t_{k}\right) \\
\geq & I_{k}\left(\int_{t_{k}-\tau_{k}}^{t_{k}-\sigma_{k}} \beta(s) d s\right)+\bar{b}_{\beta k} \\
& -\left[\int_{t_{k}-\tau_{k}}^{t_{k}-\sigma_{k}} p(s) d s+\bar{b}_{p k}, \quad k=1,2, \ldots m,\right. \\
& -L_{t_{k}-\tau_{k}}^{t_{k}-\sigma_{k}} x(s) d s+I_{k}\left(\int_{t_{k}-\tau_{k}}^{t_{k}-\sigma_{k}} q(s, x(s)) d s\right]
\end{aligned}
$$

By Lemma 3, we get $p(t) \leq 0$ on $J$; that is, $\beta \leq x$. Similar arguments show that $x \leq \alpha$.

It remains to prove that (46) possesses at least one solution. By Lemma 6, PBVP (46) is equivalent to the following impulsive integral equation:

$$
\begin{aligned}
x(t)=-\int_{0}^{T} G(t, s) & \quad \times[W x(\theta(s))+N(K x)(s)+L(S x)(s) \\
& \left.\quad+g_{q}(s)\right] d s \\
- & \sum_{k=1}^{m} G\left(t, t_{k}\right)\left[L_{k} \int_{t_{k}-\tau_{k}}^{t_{k}-\sigma_{k}} x(s) d s+e_{k}\right], \quad t \in J,
\end{aligned}
$$

where $e_{k}=I_{k}\left(\int_{t_{k}-\tau_{k}}^{t_{k}-\sigma_{k}} q(s, x(s)) d s\right)-L_{k} \int_{t_{k}-\tau_{k}}^{t_{k}-\sigma_{k}} q(s, x(s)) d s$. For any $x \in E$, define a continuous compact operator $F$ by

$$
\begin{array}{rl}
(F x)(t)=-\int_{0}^{T} & G(t, s) \\
& \times[W x(\theta(s))+N(K x)(s)+L(S x)(s)
\end{array}
$$




$$
\begin{gathered}
\left.+g_{q}(s)\right] d s \\
-\sum_{k=1}^{m} G\left(t, t_{k}\right)\left[L_{k} \int_{t_{k}-\tau_{k}}^{t_{k}-\sigma_{k}} x(s) d s+e_{k}\right], \quad t \in J .
\end{gathered}
$$

Let $\delta>0$, such that $|\alpha(t)|<\delta,|\beta(t)|<\delta, t \in J$, and take the compact sets $B=\left\{\left(t, y_{1}, y_{2}, y_{3}, y_{4}\right) \in R^{5}: t \in J, \beta(t) \leq y_{1} \leq\right.$ $\alpha(t), \beta(\theta(t)) \leq y_{2} \leq \alpha(\theta(t)),(K \beta)(t) \leq y_{3} \leq(K \alpha)(t)$, and $\left.(S \beta)(t) \leq y_{4} \leq(S \alpha)(t)\right\}$. Since $f$ is continuous, then we can choose a constant $\rho>0$, such that $\left|f\left(t, y_{1}, y_{2}, y_{3}, y_{4}\right)\right| \leq \rho$, $\left(t, y_{1}, y_{2}, y_{3}, y_{4}\right) \in B$. For $\lambda \in(0,1)$, we see that any solution of

$$
x=\lambda F x
$$

satisfies

$$
\begin{aligned}
& \|x\|_{P C}=\lambda\|F x\|_{P C} \\
& \leq \lambda \sup _{t \in J} \int_{0}^{T} G(t, s) \\
& \times[W|x(\theta(s))|+N|(K x)(s)| \\
& \left.+L|(S x)(s)|+\left|g_{q}(s)\right|\right] d s \\
& +\lambda \sum_{k=1}^{m} G\left(t, t_{k}\right)\left[L_{k} \int_{t_{k}-\tau_{k}}^{t_{k}-\sigma_{k}}|x(s)| d s+\left|e_{k}\right|\right] \\
& \leq \sup _{t \in J}\left\{\int_{0}^{T} G(t, s)\right. \\
& \times\left[W+N \int_{0}^{s} k(s, r) d r\right. \\
& \left.\left.+L \int_{0}^{T} h(s, r) d r\right] d s\right\}\|x\|_{P C} \\
& +\frac{\rho+M \delta}{M} \\
& +\sup _{t \in J} \sum_{k=1}^{m} G\left(t, t_{k}\right) \\
& \times\left[L_{k}\left(\tau_{k}-\sigma_{k}\right)\|x\|_{P C}+\left|e_{k}\right|\right] \\
& +\sup _{t \in J}\left\{\int_{0}^{T} G(t, s)\right. \\
& \times\left[W+N \int_{0}^{s} k(s, r) d r\right. \\
& \left.\left.+L \int_{0}^{T} h(s, r) d r\right] d s\right\} \delta .
\end{aligned}
$$

From the continuity of $I_{k}, k=1,2, \ldots, m$, and $\beta(t) \leq$ $q(t, x(t)) \leq \alpha(t)$ on $J$, we can choose some $\omega>0$ such that $\left|e_{k}\right|<\omega, k=1,2, \ldots, m$. Then we have

$$
\begin{gathered}
\|x\|_{P C} \leq \frac{1}{1-\mu}\left[\frac{\rho+M \delta}{M}\right. \\
+\sup _{t \in J}\left\{\int_{0}^{T} G(t, s)\right. \\
\times\left[W+N \int_{0}^{s} k(s, r) d r\right. \\
\left.\left.+L \int_{0}^{T} h(s, r) d r\right] d s\right\} \delta \\
\left.+\frac{\omega m}{1-e^{-M T}}\right],
\end{gathered}
$$

where

$$
\begin{aligned}
& \mu=\sup _{t \in J}\left\{\int_{0}^{T} G(t, s)\right. \\
& \times {\left[W+N \int_{0}^{s} k(s, r) d r\right.} \\
&\left.\left.+L \int_{0}^{T} h(s, r) d r\right] d s\right\} \\
&+\frac{1}{1-e^{-M T}} \sum_{k=1}^{m} L_{k}\left(\tau_{k}-\sigma_{k}\right)<1 .
\end{aligned}
$$

Hence, by Schaefer's theorem [21], we get that $F$ has at least a fixed point $x \in E$, which is a solution of (46). Such a solution lies between $\beta$ and $\alpha$ and, consequently, is a solution of (4). Thus, the proof is complete.

Theorem 10. Assume that there exist lower and upper solutions for PBVP (4) and assume the following.

$\left(H_{3}\right)$ The function $f \in C\left(J \times R^{4}, R\right)$ satisfies

$$
\begin{gathered}
f\left(t, z_{1}, z_{2}, z_{3}, z_{4}\right)-f\left(t, \bar{z}_{1}, \bar{z}_{2}, \bar{z}_{3}, \bar{z}_{4}\right) \\
\leq M\left(z_{1}-\bar{z}_{1}\right)+W\left(z_{2}-\bar{z}_{2}\right) \\
+N\left(z_{3}-\bar{z}_{3}\right)+L\left(z_{4}-\bar{z}_{4}\right)
\end{gathered}
$$

where $\beta(t) \leq \bar{z}_{1} \leq z_{1} \leq \alpha(t), \beta(\theta(t)) \leq \bar{z}_{2} \leq z_{2} \leq \alpha(\theta(t))$, $(K \beta)(t) \leq \bar{z}_{3} \leq z_{3} \leq(K \alpha)(t)$, and $(S \beta)(t) \leq \bar{z}_{4} \leq z_{4} \leq$ $(S \alpha)(t), t \in J$, where $M>0, W \geq 0, N \geq 0, L \geq 0$, and $\theta \in C(J, J)$.

$\left(H_{4}\right)$ The functions $I_{k} \in C(R, R)$ satisfy

$$
\begin{aligned}
& I_{k}\left(\int_{t_{k}-\tau_{k}}^{t_{k}-\sigma_{k}} x(s) d s\right)-I_{k}\left(\int_{t_{k}-\tau_{k}}^{t_{k}-\sigma_{k}} y(s) d s\right) \\
& \quad \leq L_{k} \int_{t_{k}-\tau_{k}}^{t_{k}-\sigma_{k}} x(s)-y(s) d s,
\end{aligned}
$$

where $\int_{t_{k}-\tau_{k}}^{t_{k}-\sigma_{k}} \beta(s) d s \leq \int_{t_{k}-\tau_{k}}^{t_{k}-\sigma_{k}} y(s) d s \leq \int_{t_{k}-\tau_{k}}^{t_{k}-\sigma_{k}} x(s) d s \leq$ $\int_{t_{k}-\tau_{k}}^{t_{k}-\sigma_{k}} \alpha(s) d s, k=1,2, \ldots, m$, where $L_{k} \geq 0$ and $0 \leq \sigma_{k} \leq$ $\tau_{k} \leq t_{k}-t_{k-1}, k=1,2, \ldots, m$. 
Suppose that inequalities (12) and (40) hold. Then there exist monotone sequences $\left\{\alpha_{n}\right\},\left\{\beta_{n}\right\}$ with $\alpha_{0}=\alpha, \beta_{0}=\beta$, which converge uniformly on $J$ to the extremal solutions of the periodic boundary value problem (4) in $[\beta, \alpha]$.

Proof. For any $\eta \in[\beta, \alpha]$, consider PBVP (35) with

$$
\begin{aligned}
g(t)= & f(t, \eta(t), \eta(\theta(t)),(K \eta)(t),(S \eta)(t)) \\
& -M \eta(t)-W \eta(\theta(t))-N(K \eta)(t)-L(S \eta)(t) .
\end{aligned}
$$

By Lemmas 6 and 7, PBVP (35) possesses a unique solution $x \in E$. We define an operator $A$ by $u=A \eta$; then the operator $A$ has the following properties:

(i) $\beta \leq A \beta, A \alpha \leq \alpha$;

(ii) $A \eta_{1} \leq A \eta_{2}$ for any $\eta_{1}, \eta_{2} \in[\beta, \alpha]$ with $\eta_{1} \leq \eta_{2}$.

First we prove (i). Let $p=\beta_{0}-\beta_{1}$, where $\beta_{1}=A \beta_{0}$. Then, we have $p(0)-p(T)=\beta_{0}(0)-\beta_{0}(T)$ since $\beta_{1}(0)=\beta_{1}(T)$ and

$$
\begin{aligned}
p^{\prime}(t)= & \beta_{0}^{\prime}(t)-\beta_{1}^{\prime}(t) \\
\geq & M p(t)+W p(\theta(t))+N(K p)(t) \\
& +L(S p)(t)+\bar{a}_{p}(t), \quad t \in J_{0}, \\
\Delta p\left(t_{k}\right)= & \Delta \beta_{0}\left(t_{k}\right)-\Delta \beta_{1}\left(t_{k}\right) \\
\geq & L_{k} \int_{t_{k}-\tau_{k}}^{t_{k}-\sigma_{k}} p(s) d s+\bar{b}_{p k}, \quad k=1,2, \ldots m .
\end{aligned}
$$

By Lemma 3, we get $p(t) \leq 0$ on $J$; that is, $\beta \leq A \beta$. Analogously, we have $A \alpha \leq \alpha$.

Now, we claim (ii). Set $u_{1}=A \eta_{1}, u_{2}=A \eta_{2}$, where $\eta_{1}$, $\eta_{2} \in[\beta, \alpha]$ with $\eta_{1} \leq \eta_{2}$. Let $p=u_{1}-u_{2}$; by $\left(H_{3}\right)-\left(H_{4}\right)$, we have

$$
\begin{gathered}
p^{\prime}(t)=u_{1}^{\prime}(t)-u_{2}^{\prime}(t) \\
\geq M p(t)+W p(\theta(t))+N(K p)(t) \\
+L(S p)(t), \quad t \in J_{0}, \\
\Delta p\left(t_{k}\right)=\Delta u_{1}\left(t_{k}\right)-\Delta u_{2}\left(t_{k}\right) \\
=L_{k} \int_{t_{k}-\tau_{k}}^{t_{k}-\sigma_{k}} p(s) d s, \quad k=1,2, \ldots m, \\
p(0)=p(T) .
\end{gathered}
$$

By Lemma 3 , we have $p(t) \leq 0$ on $J$ and so $u_{1} \leq u_{2}$. Thus we may define the sequences $\left\{\alpha_{n}\right\},\left\{\beta_{n}\right\}$ by $\alpha_{n+1}=A \alpha_{n}, \beta_{n+1}=$ $A \beta_{n}, \alpha_{0}=\alpha$, and $\beta_{0}=\beta$. From (i), (ii), we obtain

$$
\begin{aligned}
\beta_{0} & \leq \beta_{1} \leq \beta_{2} \leq \cdots \leq \beta_{n} \leq \cdots \\
& \leq \alpha_{n} \leq \cdots \leq \alpha_{2} \leq \alpha_{1} \leq \alpha_{0} \quad \text { on } J,
\end{aligned}
$$

and each $\alpha_{n}, \beta_{n} \in E(\forall n \in N)$ satisfies

$$
\begin{gathered}
\alpha_{n}(t)=-\int_{0}^{T} G(t, s)\left[W \alpha_{n}(\theta(s))+N\left(K \alpha_{n}\right)(s)\right. \\
\left.+L\left(S \alpha_{n}\right)(s)+g_{n-1}(s)\right] d s \\
-\sum_{k=1}^{m} G\left(t, t_{k}\right)\left[L_{k} \int_{t_{k}-\tau_{k}}^{t_{k}-\sigma_{k}} \alpha_{n}(s) d s\right. \\
+I_{k}\left(\int_{t_{k}-\tau_{k}}^{t_{k}-\sigma_{k}} \alpha_{n-1}(s) d s\right) \\
\left.-L_{k} \int_{t_{k}-\tau_{k}}^{t_{k}-\sigma_{k}} \alpha_{n-1}(s) d s\right], \quad t \in J, \\
\beta_{n}(t)=-\int_{0}^{T} G(t, s)\left[W \beta_{n}(\theta(s))+N\left(K \beta_{n}\right)(s)\right. \\
\left.+L\left(S \beta_{n}\right)(s)+\bar{g}_{n-1}(s)\right] d s \\
-\sum_{k=1}^{m} G\left(t, t_{k}\right)\left[L_{k}^{t_{k}-\sigma_{k}} \beta_{t_{k}-\tau_{k}}(s) d s\right. \\
+I_{k}\left(\int_{t_{k}-\tau_{k}}^{t_{k}-\sigma_{k}} \beta_{n-1}(s) d s\right) \\
\left.-L_{k} \int_{t_{k}-\tau_{k}}^{t_{k}-\sigma_{k}} \beta_{n-1}(s) d s\right], \quad t \in J,
\end{gathered}
$$

where

$$
\begin{aligned}
g_{n-1}(t) \\
=f\left(t, \alpha_{n-1}(t), \alpha_{n-1}(\theta(t)),\left(K \alpha_{n-1}\right)(t),\left(S \alpha_{n-1}\right)(t)\right) \\
\quad-M \alpha_{n-1}(t)-W \alpha_{n-1}(\theta(t)) \\
\quad-N\left(K \alpha_{n-1}\right)(t)-L\left(S \alpha_{n-1}\right)(t), \\
\bar{g}_{n-1}(t) \\
=f\left(t, \beta_{n-1}(t), \beta_{n-1}(\theta(t)),\left(K \beta_{n-1}\right)(t),\left(S \beta_{n-1}\right)(t)\right) \\
-M \beta_{n-1}(t)-W \beta_{n-1}(\theta(t)) \\
-N\left(K \beta_{n-1}\right)(t)-L\left(S \beta_{n-1}\right)(t) .
\end{aligned}
$$

Hence, there exist $\xi, \psi$ such that $\lim _{n \rightarrow \infty} \alpha_{n}(t)=\psi(t)$ and $\lim _{n \rightarrow \infty} \beta_{n}(t)=\xi(t)$ uniformly on $J$. Clearly, $\xi, \psi$ satisfy PBVP (4). We will prove that $\xi, \psi$ are extreme solutions of PBVP (4). Let $x(t)$ be any solution of PBVP (4), which satisfies $\beta(t) \leq x(t) \leq \alpha(t), t \in J$. Also suppose there exists a positive integer $n$ such that for $t \in J, \beta_{n}(t) \leq x(t) \leq \alpha_{n}(t)$. Setting $p(t)=\beta_{n+1}(t)-x(t)$, then for $t \in J$,

$$
\begin{aligned}
p^{\prime}(t) & =\beta_{n+1}^{\prime}(t)-x^{\prime}(t) \\
\geq & M p(t)+W p(\theta(t))+N(K p)(t)+L(S p)(t), \\
& t \in J_{0},
\end{aligned}
$$




$$
\begin{aligned}
\Delta p\left(t_{k}\right)= & \Delta \beta_{n+1}\left(t_{k}\right)-\Delta x\left(t_{k}\right) \\
\geq & L_{k} \int_{t_{k}-\tau_{k}}^{t_{k}-\sigma_{k}} p(s) d s, \\
& k=1,2, \ldots m, \quad p(0)=p(T) .
\end{aligned}
$$

According to Lemma 3, we get that $p(t) \leq 0$ on $J$. Similarly, we obtain $x(t) \leq \alpha_{n+1}(t)$ on $J$. Since $\beta_{0} \leq x(t) \leq \alpha_{0}(t)$ on $J$, by induction, we have $\beta_{n}(t) \leq x(t) \leq \alpha_{n}(t)$ on $J$ for all $n$. Therefore, $\xi(t) \leq x(t) \leq \psi(t)$ on $J$ by taking limit as $n \rightarrow \infty$. The proof is complete.

\section{An Example}

In this section, in order to illustrate our results, we consider an example.

Example 11. Consider the following periodic boundary value problem:

$$
\begin{gathered}
u^{\prime}(t)=\frac{1}{6} t^{2}(1+u(t))+\frac{1}{16} t u\left(\frac{1}{2} t\right)+\frac{1}{36}\left[\int_{0}^{t} t s^{3} u(s) d s\right]^{3} \\
+\frac{1}{24}\left[\int_{0}^{1} t^{3} s u(s) d s\right]^{2}, \quad t \in J=[0,1], t \neq \frac{1}{2} \\
\Delta u\left(\frac{1}{2}\right)=\frac{1}{2} \int_{1 / 6}^{1 / 4} u(s) d s, \quad k=1, \quad u(0)=u(1)
\end{gathered}
$$

where $k(t, s)=t s^{3}, h(t, s)=t^{3} s, m=1, t_{1}=1 / 2, \sigma_{1}=1 / 4$, $\tau_{1}=1 / 3$, and $T=1$.

Obviously, $\alpha_{0}=0, \beta_{0}=-5$ are lower and upper solutions for (64), respectively, and $\beta_{0} \leq \alpha_{0}$.

Let

$$
f\left(t, z_{1}, z_{2}, z_{3}, z_{4}\right)=\frac{1}{6} t^{2}\left(1+z_{1}\right)+\frac{1}{16} t z_{2}+\frac{1}{36} z_{3}^{3}+\frac{1}{24} z_{4}^{2} \text {. }
$$

We have

$$
\begin{aligned}
& f\left(t, z_{1}, z_{2}, z_{3}, z_{4}\right)-f\left(t, \bar{z}_{1}, \bar{z}_{2}, \bar{z}_{3}, \bar{z}_{4}\right) \\
& \quad \leq \frac{1}{6}\left(z_{1}-\bar{z}_{1}\right)+\frac{1}{16}\left(z_{2}-\bar{z}_{2}\right)+\frac{1}{3}\left(z_{3}-\bar{z}_{3}\right)+\frac{1}{4}\left(z_{4}-\bar{z}_{4}\right),
\end{aligned}
$$

where $\beta(t) \leq \bar{z}_{1} \leq z_{1} \leq \alpha(t), \beta((1 / 2) t) \leq \bar{z}_{2} \leq z_{2} \leq$ $\alpha((1 / 2) t),(K \beta)(t) \leq \bar{z}_{3} \leq z_{3} \leq(K \alpha)(t)$, and $(S \beta)(t) \leq \bar{z}_{4} \leq$ $z_{4} \leq(S \alpha)(t), t \in J$. It is easy to see that

$$
\begin{aligned}
& I_{1}\left(\int_{1 / 6}^{1 / 4} x(s) d s\right)-I_{1}\left(\int_{1 / 6}^{1 / 4} y(s) d s\right) \\
& \quad=\frac{1}{2} \int_{1 / 6}^{1 / 4} x(s)-y(s) d s,
\end{aligned}
$$

where $\int_{t_{k}-\tau_{k}}^{t_{k}-\sigma_{k}} \beta(s) d s \leq \int_{t_{k}-\tau_{k}}^{t_{k}-\sigma_{k}} y(s) d s \leq \int_{t_{k}-\tau_{k}}^{t_{k}-\sigma_{k}} x(s) d s \leq$ $\int_{t_{k}-\tau_{k}}^{t_{k}-\sigma_{k}} \alpha(s) d s, k=1$.

Taking $L_{1}=1 / 2, M=1 / 6, W=1 / 16, N=1 / 3$, and $L=1 / 4$, it follows that

$$
\begin{aligned}
\widehat{L}= & L_{k}\left(\tau_{k}-\sigma_{k}\right)=\frac{1}{24} \\
r(t)= & M+W+N \int_{0}^{t} k(t, s) d s+L \int_{0}^{T} h(t, s) d s \\
= & \frac{1}{6}+\frac{1}{16}+\frac{1}{3} \int_{0}^{t} t s^{3} d s+\frac{1}{4} \int_{0}^{1} t^{3} s d s \\
A_{k}= & +L_{k}\left(\tau_{k}-\sigma_{k}\right)=1+\frac{1}{2}\left(\frac{1}{3}-\frac{1}{4}\right)=\frac{25}{24}, \\
B_{k}= & A_{k} \int_{t_{k-1}}^{t_{k}-\tau_{k}} r(s) d s \\
& +\int_{t_{k}-\tau_{k}}^{t_{k}-\sigma_{k}}\left[1+L_{k}\left(t_{k}-\sigma_{k}-s\right)\right] r(s) d s \\
& +\int_{t_{k}-\sigma_{k}}^{t_{k}} r(s) d s, \\
& +\int_{(1 / 2)-(1 / 4)}^{1 / 2} r(s) d s . \\
& +\int_{(1 / 2)-(1 / 3)}^{(1 / 2)-(1 / 4)}\left[1+\frac{1}{2}\left(\frac{1}{2}-\frac{1}{4}-s\right)\right] r(s) d s \\
= & A_{k}(s) d s
\end{aligned}
$$

Thus,

$$
\begin{gathered}
\left(\widehat{L}+\sum_{k=1}^{m} B_{k}+\int_{0}^{T} r(s) d s\right) \prod_{k=1}^{m} A_{k} \approx 0.4528309326 \leq 1, \\
\sup _{t \in J}\left\{\int _ { 0 } ^ { T } G ( t , s ) \left(W+N \int_{0}^{s} k(s, r) d r\right.\right. \\
\left.\left.+L \int_{0}^{T} h(s, r) d r\right) d s\right\} \\
+\frac{1}{1-e^{-M T}} \sum_{k=1}^{m} L_{k}\left(\tau_{k}-\sigma_{k}\right) \\
=\sup _{t \in J}\left\{\int _ { 0 } ^ { 1 } G ( t , s ) \left(\frac{1}{16}+\frac{1}{3} \int_{0}^{s} s r^{3} d r\right.\right. \\
+\frac{1}{1-e^{-1 / 6}} \frac{1}{2}\left(\frac{1}{3}-\frac{1}{4}\right) \approx 0.9256866285<1
\end{gathered}
$$


Therefore, (64) satisfies all conditions of Theorem 10. So PBVP (64) has minimal and maximal solutions in the segment $\left[\beta_{0}, \alpha_{0}\right]$.

\section{Conflict of Interests}

The authors declare that there is no conflict of interests regarding the publication of this paper.

\section{Acknowledgments}

This research is supported by the Centre of Excellence in Mathematics, the Commission on Higher Education, Thailand, and King Mongkut's University of Technology North Bangkok, Thailand.

\section{References}

[1] D. D. Bainov and P. S. Simeonov, Impulsive Differential Equations: Asymptotic Properties of the Solutions, vol. 28 of Series on Advances in Mathematics for Applied Sciences, World Scientific, Singapore, 1995.

[2] D. D. Bainov and P. S. Simeonov, Impulsive Differential Equations: Periodic Solutions and Applications, Longman Scientific and Technical, Harlow, UK, 1993.

[3] M. Benchohra, J. Henderson, and S. Ntouyas, Impulsive Differential Equations and Inclusions, vol. 2 of Contemporary Mathematics and Its Applications, Hindawi Publishing Corporation, New York, NY, USA, 2006.

[4] V. Lakshmikantham, D. D. Bainov, and P. S. Simeonov, Theory of Impulsive Differential Equations, vol. 6 of Series in Modern Applied Mathematics, World Scientific, Singapore, 1989.

[5] A. M. Samoilenko and N. A. Perestyuk, Impulsive Differential Equations, World Scientific, Singapore, 1995.

[6] G. S. Ladde, V. Lakshmikantham, and A. S. Vatsala, Monotone Iterative Techniques for Nonlinear Differential Equations, Advanced Texts and Surveys in Pure and Applied Mathematics, Pitman, Boston, Mass, USA, 1985.

[7] B. Ahmad and J. J. Nieto, "Existence and approximation of solutions for a class of nonlinear impulsive functional differential equations with anti-periodic boundary conditions," Nonlinear Analysis: Theory, Methods \& Applications, vol. 69, no. 10, pp. 3291-3298, 2008.

[8] L. Chen and J. Sun, "Nonlinear boundary value problem for first order impulsive integro-differential equations of mixed type," Journal of Mathematical Analysis and Applications, vol. 325, no. 2, pp. 830-842, 2007.

[9] X. He, J. Xie, G. Chen, and J. Shen, "Integral BVPs for a class of first-order impulsive functional differential equations," International Journal of Differential Equations, vol. 2010, Article ID 908960, 11 pages, 2010.

[10] T. Jankowski, "First-order impulsive ordinary differential equations with advanced arguments," Journal of Mathematical Analysis and Applications, vol. 331, no. 1, pp. 1-12, 2007.

[11] J. Li and J. Shen, "New comparison results for impulsive functional differential equations," Applied Mathematics Letters, vol. 23, no. 4, pp. 487-493, 2010.

[12] Z. Luo and J. J. Nieto, "New results for the periodic boundary value problem for impulsive integro-differential equations," Nonlinear Analysis: Theory, Methods \& Applications, vol. 70, no. 6, pp. 2248-2260, 2009.
[13] G. Wang, L. Zhang, and G. Song, "New existence results and comparison principles for impulsive integral boundary value problem with lower and upper solutions in reversed order," Advances in Difference Equations, vol. 2011, Article ID 783726, 2011.

[14] X. Yang and J. Shen, "Nonlinear boundary value problems for first order impulsive functional differential equations," Applied Mathematics and Computation, vol. 189, no. 2, pp. 1943-1952, 2007.

[15] L. Zhang, "Boundary value problem for first order impulsive functional integro-differential equations," Journal of Computational and Applied Mathematics, vol. 235, no. 8, pp. 2442-2450, 2011.

[16] Z. Liu, J. Han, and L. Fang, "Integral boundary value problems for first order integro-differential equations with impulsive integral conditions," Computers \& Mathematics with Applications, vol. 61, no. 10, pp. 3035-3043, 2011.

[17] Z. Liu, J. Han, and L. Fang, "Nonlinear boundary value problems for first order integro-differential equations with impulsive integral conditions," Bulletin of the Malaysian Mathematical Sciences Society, vol. 36, no. 2, pp. 435-446, 2013.

[18] J. Tariboon, "Boundary value problems for first order functional differential equations with impulsive integral conditions," Journal of Computational and Applied Mathematics, vol. 234, no. 8, pp. 2411-2419, 2010.

[19] C. Thaiprayoon, D. Samana, and J. Tariboon, "Multi-point boundary value problem for first order impulsive integrodifferential equations with multi-point jump conditions," Boundary Value Problems, vol. 2012, article 38, 2012.

[20] P. Thiramanus and J. Tariboon, "Impulsive differential and impulsive integral inequalities with integral jump conditions," Journal of Inequalities and Applications, vol. 2012, article 25, 2012.

[21] D. R. Smart, Fixed Point Theorems, Cambridge University Press, Cambridge, UK, 1980. 


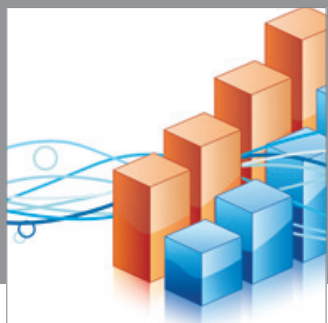

Advances in

Operations Research

mansans

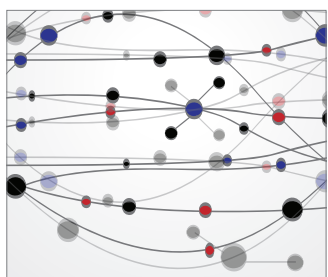

The Scientific World Journal
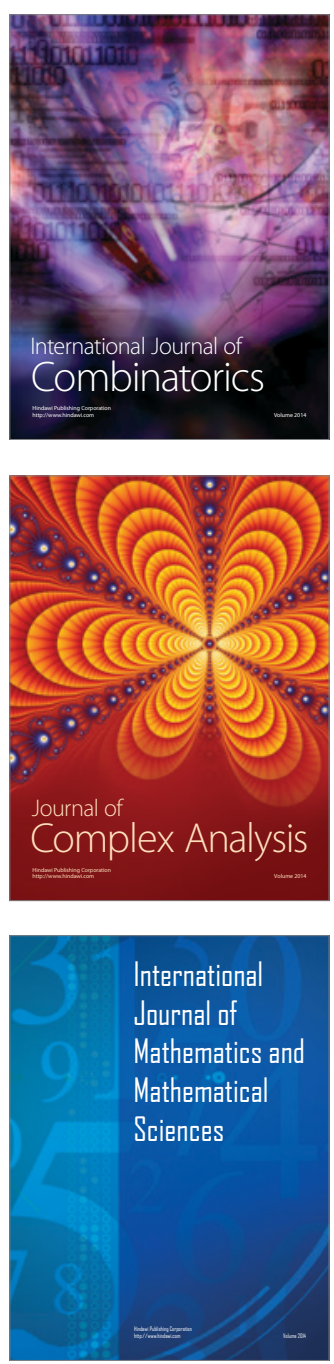
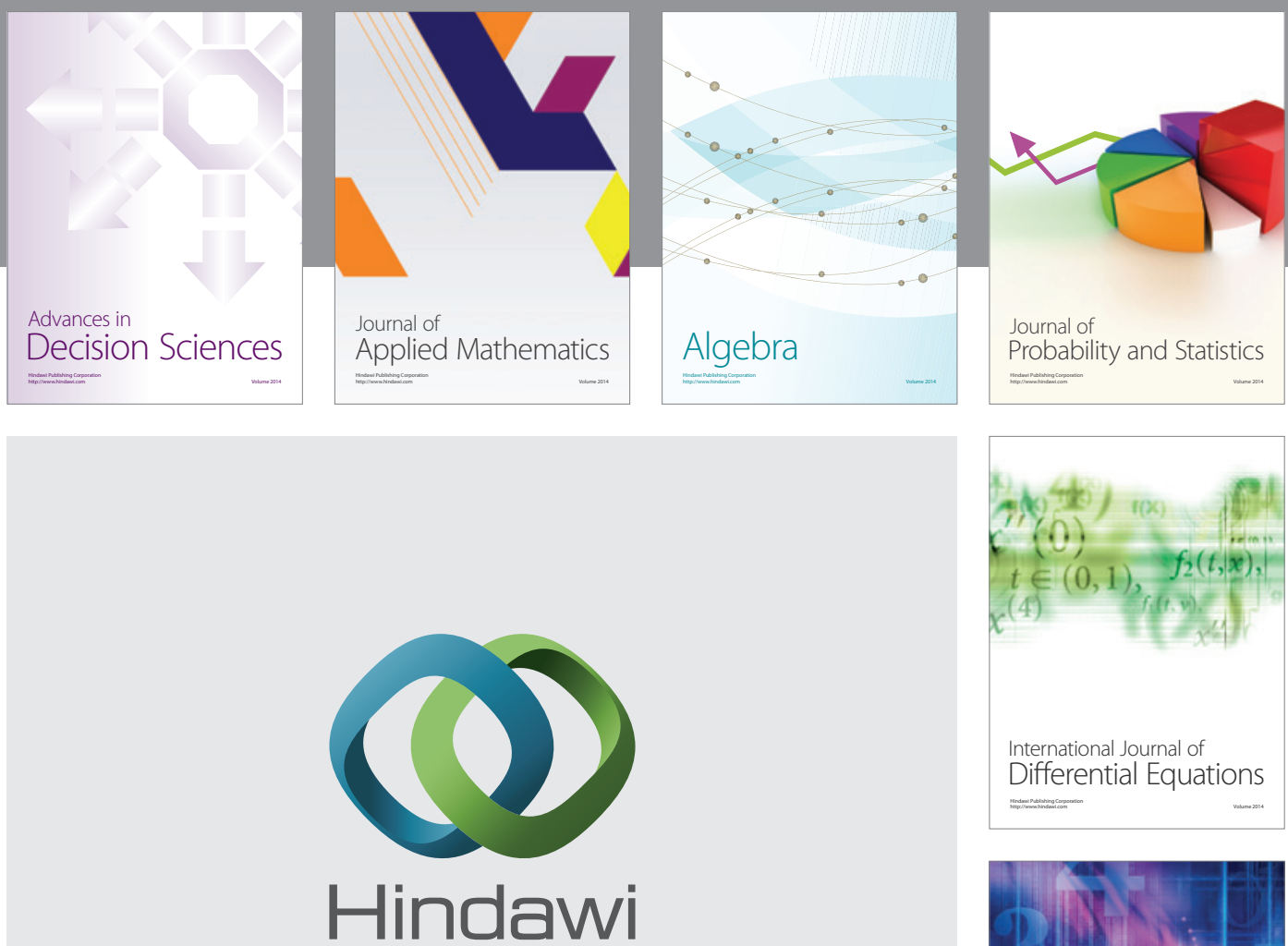

Submit your manuscripts at http://www.hindawi.com
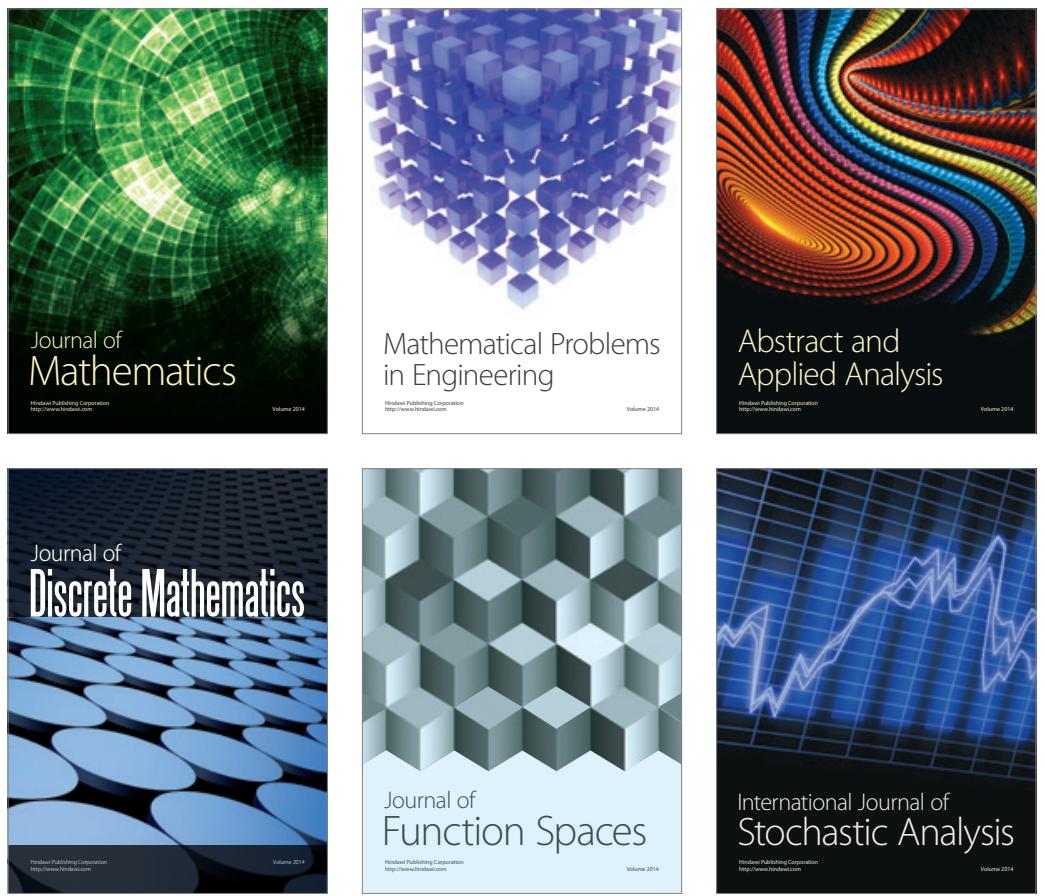

Journal of

Function Spaces

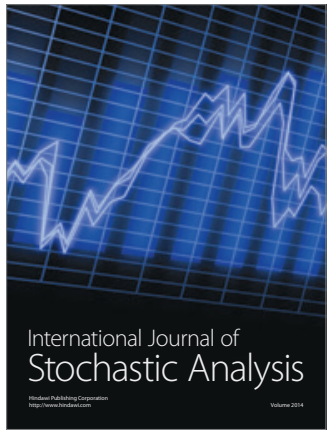

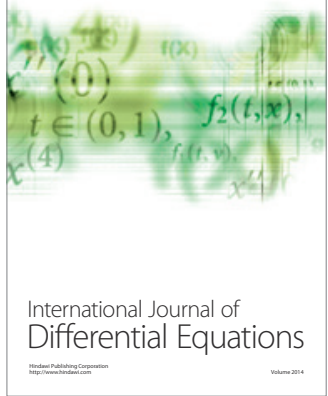
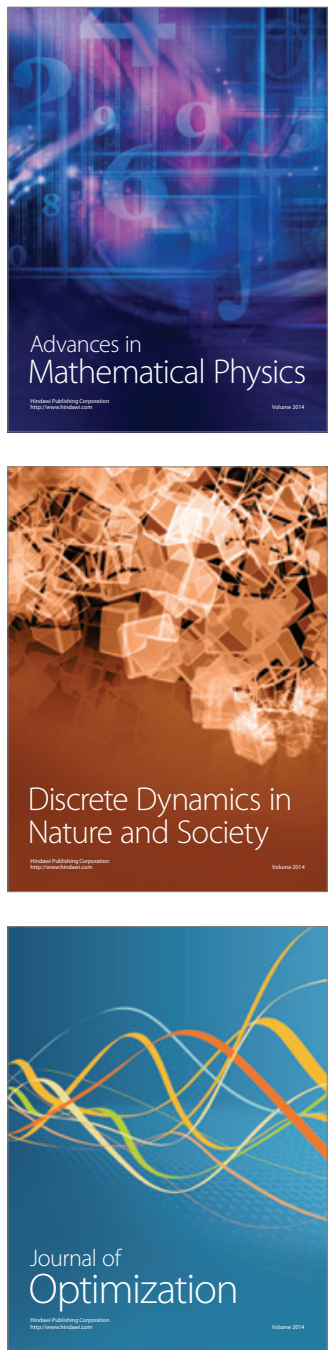